\title{
LV-VE-1
}

\section{Laparoscopic resection of segment 4 and ventral area of anterior section in cirrhotic liver}

\author{
Yang Won $\mathrm{NAH}^{*, 1}$, Sang Min KONG ${ }^{1}$, Eun Ji LEE ${ }^{1}$, Jun Ho JUNG ${ }^{2}$ \\ 'Department of Surgery, Ulsan University Hospital, Ulsan, Korea \\ ${ }^{2}$ Department of Internal Medicine, Ulsan University Hospital, Ulsan, Korea
}

Introduction: In this video, I will describe the laparoscopic procedure where the hepatic territory supplied by the Glisson pedicles to the segment 4 and the ventral branches of the right anterior Glisson pedicle is resected.

Methods: A 48 years old male patient presented with two HCC's. Chronic hepatits B was diagnosed 20 years ago. 8 months ago a hypoechoic lesion $(1.5 \mathrm{~cm}$ ) was identified in right liver. No treatment was given. On follow CT scan, 2 HCC's were identified. One tumor was $1.8 \mathrm{~cm}$ in size and located at segment $5-6,2 \mathrm{~cm}$ deep from the liver surface. The other tumor was $1.5 \mathrm{~cm}$ in size and located at liver hilum, in between the left and right anterior Glisson pedicles and in proximity to the middle hepatic vein. The level of AFP was $3.7 \mathrm{ng} / \mathrm{mL}$ and PIVKA II $126 \mathrm{mAU} / \mathrm{mL}$. E was $10.4 \mathrm{kPa}$ on fibroscan. For the sake of preservation of liver volume, the former lesion was ablated with radiofrequency energy before the surgery. And for the latter lesion, laparoscopic resection of segment 4 and ventral area of anterior section was performed.

Results: The operation took 390 minutes. No blood product was given perioperatively. The patient was discharged 6 days after the operation without any complication. He is now tumor free 8 months after the operation.

Conclusions: Laparoscopic resection of segment 4 and ventral area of anterior section in cirrhotic liver can be performed safely and anatomically. Tumor adherent to the middle hepatic vein is a good indication for this parenchyma-sparing resection. 